\title{
A patient with rapid cognitive decline and visual failure
}

Thashi Chang

Department of Clinical Medicine, Faculty of Medicine, University of Colombo

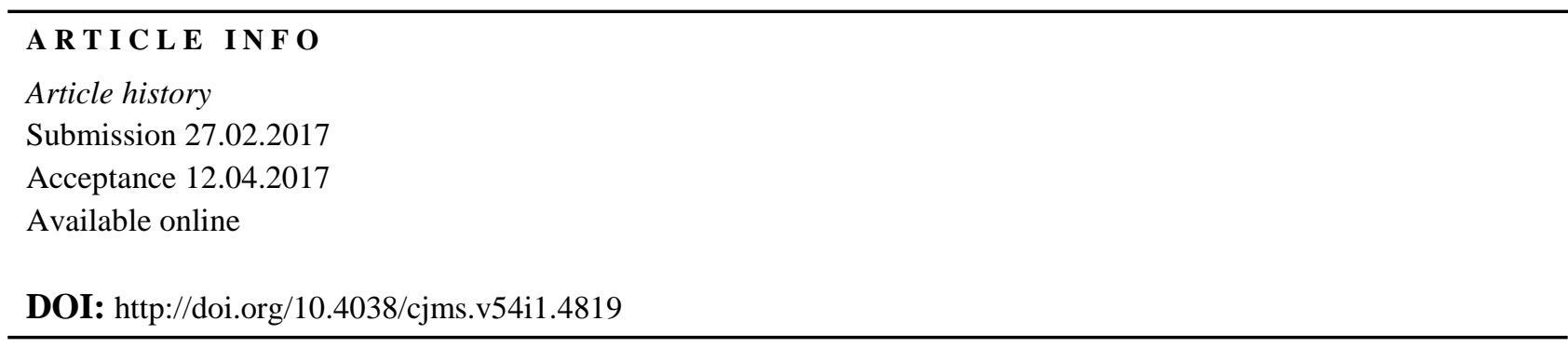

\section{Case vignette}

A previously healthy 63-year-old man was brought to the psychiatrist because of increasingly becoming withdrawn over a period of one month. He was noted to be cognitively impaired and blind and thus referred to the neurologist. His pupils were normal in size and reaction to light, and the optic fundi were normal. He was noted to have symmetrical bradykinesia and rigidity in his limbs. Diffusion-weighted magnetic resonance image (DWI) of his brain and part of his electroencephalogram (EEG) record are shown.

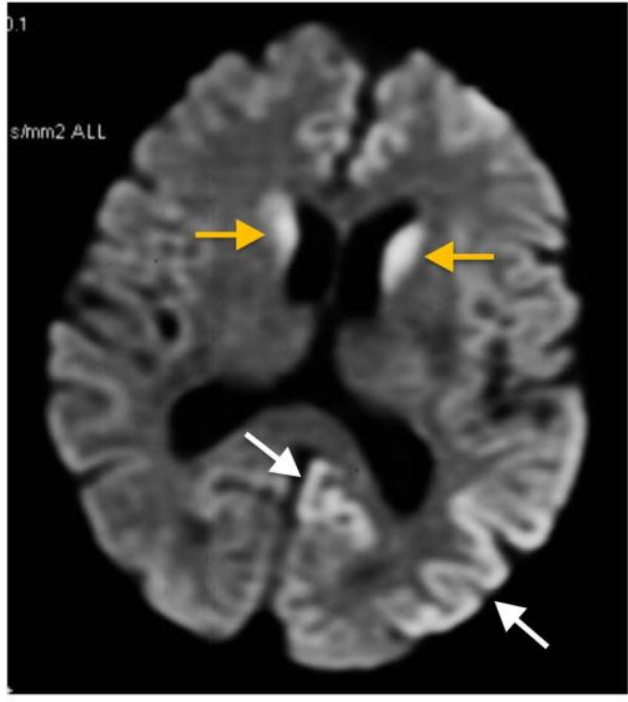

DWI brain

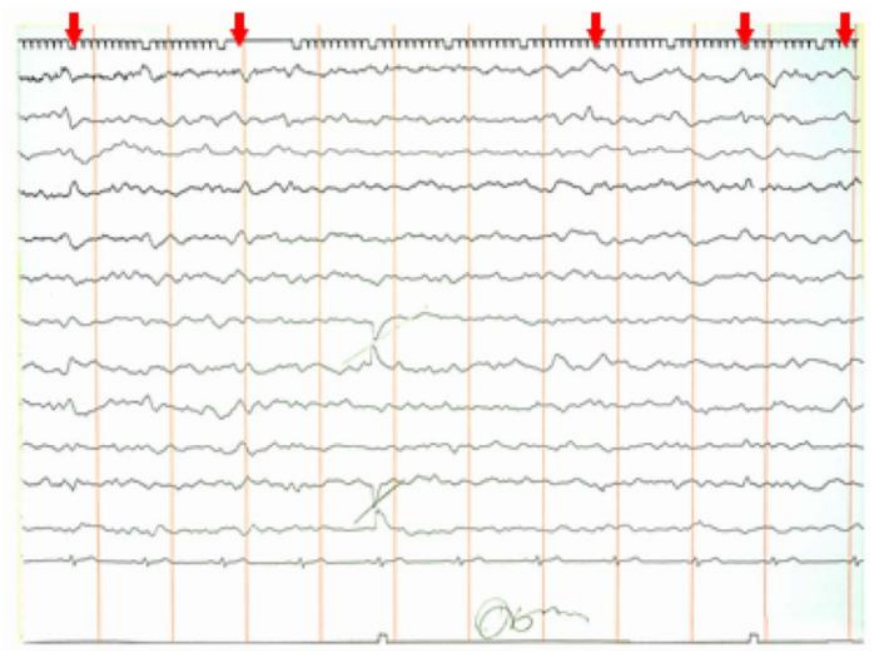

EEG

\section{Q: What is the probable diagnosis?}

\section{Corresponding Author:}

Thashi Chang

MBBS, MD, MRCP(UK), MRCP(UK)(Neurology), FCCP,

$\operatorname{FRCP}$ (Lond), DPhil(Oxon)

Department of Clinical Medicine

Faculty of Medicine - University of Colombo

25, Kynsey Road, Colombo 00800, Sri Lanka

thashichang@gmail.com

(iD) http://orcid.org/0000-0002-6027-7883

This is an open-access article distributed under the terms of the Creative Commons Attribution License, which permits unrestricted use distribution, and reproduction in any medium, provided the original author and source are credited. 


\section{A:Sporadic Creutzfeldt-Jakob disease}

Prion diseases are rare neurodegenerative diseases, which progress inexorably resulting in fatality. Creutzfeldt-Jakob disease (CJD) is the most frequent of the human prion diseases. Sporadic (sCJD), familial (fCJD), iatrogenic (iCJD), and variant forms of CJD (vCJD) are recognized, but the majority of CJD cases are sporadic $(85-95 \%)$ [1].

Although brain biopsy remains the gold standard diagnostic test for SCJD, a probable diagnosis can be established based on the following diagnostic criteria [2]:

- Progressive dementia and

- At least two out of the following four clinical features: myoclonus; visual or cerebellar disturbance; pyramidal/extrapyramidal dysfunction; akineticmutism and

- Atypical EEG during an illness of any duration, and/or a positive 14-3-3 cerebrospinal fluid (CSF) assay with a clinical duration to death less than two years, and/or high signal abnormalities in caudate nucleus and/or putamen on diffusionweighted magnetic resonance imaging (MRI) or fluid attenuated inversion recovery (FLAIR) and

- Routine investigations should not suggest an alternative diagnosis.

This patient presented with rapidly progressive cognitive decline that was recognized by the family as being increasingly withdrawn associated with cortical blindness, which presents as blindness with intact anterior visual pathways. Although myoclonus is present in more than 90 percent of patients at some point during the illness, it may be absent at presentation, even when dementia is profound as was the case in this patient.

The MRI in SCJD is characterized by increased DWI signal in the cortex ('cortical ribboning') or deep gray matter (particularly the caudate nucleus and anterior putamen) or both as was seen in this patient (white arrows). DWI of mitochondrial encephalopathy, lactic acidosis, and stroke-like episodes (MELAS); Wilson disease; cerebral anoxia; hypoglycaemia; and Wernicke encephalopathy can demonstrate similar abnormalities. However, the clinical course and laboratory findings easily distinguish them from CJD. Periodic synchronous bi- or tri-phasic sharp wave complexes on EEG are highly suggestive, but not definitive of sCJD (red arrows). Detection of 14-3-3 protein in CSF is an adjunctive rather than absolute test for the diagnosis of SCJD.

\section{Learning points}

- Rapidly progressive cognitive decline and myoclonus are the two cardinal clinical manifestations of sCJD. However, sCJD can present without myoclonus. Associated cortical blindness, extrapyramidal and cerebellar signs should suggest the diagnosis.

- A typical clinical presentation with corroborating findings on MRI and EEG are often sufficient to exclude other causes and establish SCJD as the probable diagnosis.

- Cranial MRI is mandatory in patients presenting with rapid cognitive decline. DWI is the most sensitive MRI technique, which can detect CJDrelated lesions even before the onset of symptoms. Computerized tomography (CT) is not sensitive in detecting cerebral abnormalities of CJD.

\section{References}

1. Ladogana A, Puopolo M, Croes EA, Budka H, Jarius C, Collins S, et al. Mortality from Creutzfeldt-Jakob disease and related disorders in Europe, Australia, and Canada. Neurology. 2005;64(9):1586.

2. CDC's Diagnostic Criteria for Creutzfeldt-Jakob Disease (CJD), 2010 (last updated in February 2015) http://www.cdc.gov/ncidod/dvrd/cjd/diagnostic_ criteria.html (Accessed on February 24, 2017). 